\title{
On local observer design for LQR problems with tracking
}

\author{
Paolo Di Giamberardino ${ }^{10000-0002-9113-8608]}$ and Daniela \\ Iacoviello ${ }^{1[0000-0003-3506-1455]}$ \\ Dept. Computer, Control and Management Engineering Antonio Ruberti, \\ Sapienza University of Rome, Rome, Italy \\ paolo.digiamberardino@uniroma1.it \\ https://www.diag.uniroma1.it/digiamb/website \\ daniela.iacoviello@uniroma1.it \\ https://www.diag.uniroma1.it/iacoviel
}

\begin{abstract}
The paper addresses the problem of an observer design for a nonlinear system for which a linear approach is followed for the control synthesis. The linear context driven by the control design allows to focus the observers design in the class of local, i.e. linear, observers. It is shown that when the control contains an external reference, the solution obtained working with the linear approximation to get local solutions produces non consistent results in terms of local regions of convergence for the system and for the observer. The case of a control law which solves a LQR problem with tracking is addressed and two different approaches with respect to the classical one for the observer design are studied. The results are applied to an epidemic spread control to check the differences in the performances for the two different approaches.
\end{abstract}

Keywords: Nonlinear Systems - Linear Observer · Optimal Control · LQR · Epidemic Spread.

\section{Introduction}

The problem of the state measure for dynamical systems plays an important role in control theory since state feedback solutions are often obtained in the design procedures. When the state of a dynamics is not measurable, the design of a state observer becomes a necessary step. The history of solutions to this problem begins with the case of linear dynamics [9] and, less than ten years later, it is enriched with the first results for nonlinear ones.

Several solutions have been presented in literature for the design of state observers, many of them, especially in the more recent literature, dealing with the case of nonlinear dynamics, for which nonlinear solutions have been proposed.

Some of the results are represented by nonlinear solutions which mainly follows the idea initially proposed in [9] for linear systems: an observer can be designed starting from a copy of the dynamics with corrective terms added to get the stabilization of the linear approximation of the observer and of the full 
interconnected system. Examples are [2], [16] and [15], where autonomous dynamics are considered. The importance of starting with a local solution is usually put in evidence separating explicitly the linear component of the system from the remaining nonlinear terms, to better highlight the local behaviours, [8]. A further example of a solution based on the possibility of linearising the error dynamics is represented by [12].

The explicit presence of the input in the nonlinear dynamics may complicate the approaches, since suitable bounding conditions must be given; a different solution can be obtained modelling the input as the output of an exosystem with known structure [14]. The list of references could be very long, till nowadays with, for example, [13], where an approximated linearising feedback for the system dynamics is introduced. When observers are part of a feedback control schemes for systems with non measurable state, the control design and the observer determinations are two problems that must be solved at the same time. In the linear case, where the Separation Principle holds, it is possible to design the state feedback control and the observer separately, since it can be proved that the addition of an observer in the control scheme does not change the dynamical characteristics of the controlled system. In the nonlinear case, in general the Separation Principle does not hold but can be invoked once local liner approximations are considered, so restricting to local solutions.

This paper addresses the problem introduced in the local observer design for a nonlinear system when the nominal state feedback introduced changes the equilibrium point of the controlled system. In this case, an ambiguity arises since the concept of local for the control and for the observer refers to two different equilibrium points: the initial one, considered for the linearised control design, and the new one, the equilibrium for the controlled dynamics and then the actual working point. The classical approach refers to the computation of local solutions referring to the open loop equilibrium point; it always works for linear systems, and works in nonlinear contest when the control does not change such a point. When the control law contains a tracking term, the equilibrium point changes and the meaning of local must be discussed. A tracking term always appears when a LQR problem with tracking term is defined for the control law design. This is the case addressed in the paper to better define the class of problems considered. Two possible approaches to the control and the observer design are reported. The first one aims at working in the new equilibrium point for the controlled dynamics and the local solution for the observer design are referred to such a point. The second one tries to introduce the observer design at the same time as the control one, changing the LQR problem formulation.

Since this paper is an extended version of [7] presented at the ICINCO2019 conference, the first approach is introduced and described in the cited paper, along with the discussion of some simulation results, and is here shortly recalled. The extension is represented by the introduction of the second approach which, after its presentation, discussion and implementation on the same case study adopted in [7], is compared to the previous one. 
The proposed procedures are applied to a case study, represented by the control of an epidemic spread of a virus, the one responsible of HIV/AIDS infections, to compare their performances. The model proposed in $[3,4]$ is adopted and, following [5], the control problem is formulated in the framework of optimal control theory introducing a quadratic cost index.

In Section 2, after the general problem definition, the differences between a linear and nonlinear cases introduced by the tracking term are illustrated. A first approach to overcome such a problem is presented and discussed in Section 3 while a different solution obtained by changing the problem definition, designing the controller and the observer together, is discussed in Section 4. The procedure is then applied to the case study in Section 5 for comparative purpose and some results of numerical simulations are reported in Section 6 to validate the proposed solution. Concluding remarks in Section 7 end the paper.

\section{Problem Definition}

Given the nonlinear dynamics

$$
\begin{aligned}
\dot{x} & =f(x)+g(x) u \\
y & =h(x)
\end{aligned}
$$

with $x \in \Re^{n}, u \in \Re^{m}, y \in \Re^{p}$, and one equilibrium point $x_{e}\left(f\left(x_{e}\right)=0\right.$, $g\left(x_{e}\right) \neq 0, h\left(x_{e}\right)=0$ ), define an optimal control problem introducing the cost function

$$
J=\int_{0}^{\infty}\left(x^{T} Q x+u^{T} R u\right) d t
$$

to be minimised. Despite the nonlinearity of the system, the quadratic form in (3) suggests that if the dynamics were linear, the problem would be solved using a classical LQR design, with the additional advantage to obtain a closed loop state feedback solution. Then, the idea followed in [5] is to linearise the dynamics in a neighbourhood of one equilibrium point and solve the problem in a first order approximated way. The linear approximation of (1)-(2) can be computed, getting

$$
\begin{aligned}
\dot{\tilde{x}} & =A \tilde{x}+B u \\
\tilde{y} & =C \tilde{x}
\end{aligned}
$$

where, as usual, $\tilde{x}=x-x_{e}$ and

$$
A=\left.\frac{\partial f}{\partial x}\right|_{x=x_{e}}, \quad B=g\left(x_{e}\right), \quad C=\left.\frac{\partial h}{\partial x}\right|_{x=x_{e}}
$$

In order to have homogeneous expressions in the optimal control problem, once the local referred state variable $\tilde{x}=x-x_{e}$ is introduced, the cost function to be minimised must be rewritten as

$$
J=\int_{0}^{\infty}\left(\left(\tilde{x}+x_{e}\right)^{T} Q\left(\tilde{x}+x_{e}\right)+u^{T} R u\right) d t
$$


The problem can be faced as a LQR problem with a reference term. Classical theory shows that the stationary Riccati equation can be introduced,

$$
K B R^{-1} B^{T} K-K A-A^{T} K-Q=0
$$

whose solution $K$ gives the optimal control

$$
u=-R^{-1} B^{T} K \tilde{x}-R^{-1} B^{T}\left(K B R^{-1} B^{T}-A^{T}\right)^{-1} Q x_{e}
$$

The form of the feedback control (8) is the same as the one considered in [7],

$$
u=F \tilde{x}+r
$$

The linear term $F \tilde{x}$ satisfies the local stability of the controlled system in a neighbourhood of the equilibrium point. The additional presence of a forcing constant term $r$ in (9) is present.

In this case, the closed loop dynamics under state measurement becomes

$$
\dot{\tilde{x}}=(A+B F) \tilde{x}+B r
$$

As performed in $[5,7]$, the necessity of a state observer is considered. The procedure followed in [5] used a classical Luenberger linear state observer [9]

$$
\dot{\tilde{z}}=(A-G C) \tilde{z}+B u+G y
$$

designed on the basis of the linear approximated dynamics (4) for which the optimal state feedback (8) has been designed. The control law (8) becomes

$$
u=F \tilde{z}+r
$$

The consequent dynamics of the error $e=\tilde{z}-\tilde{x}$, locally in a neighbourhood of $x_{e}$, is described by

$$
\begin{aligned}
\dot{e} & =(A-G C) \tilde{z}+B u+G y-A \tilde{x}-B u= \\
& =(A-G C) \tilde{z}+G C \tilde{x}-A \tilde{x}=(A-G C) e
\end{aligned}
$$

asymptotically convergent to zero once $\sigma(A-G C) \in C^{-}$. Then, the asymptotic condition $\lim _{t \rightarrow \infty}\|\tilde{z}-\tilde{x}\|=0$ holds and it can be rewritten as $\lim _{t \rightarrow \infty}\|\tilde{z}-\tilde{x}\|=$ $\lim _{t \rightarrow \infty}\left\|\tilde{z}+x_{e}-x\right\|=0$ showing that if $\tilde{z}$ is the estimate of $\tilde{x}$, then $z=\tilde{z}+x_{e}$ is the estimate of the original state $x$.

Remaining in the approximated context, the whole system obtained using the state reconstructed by the observer in the control law, (12), is described by

$$
\begin{aligned}
& \dot{\tilde{x}}=A \tilde{x}+B F \tilde{z}+B r \\
& \dot{\tilde{z}}=(A-G C) \tilde{z}+B F \tilde{z}+B r+G C \tilde{x}
\end{aligned}
$$

and, replacing the observer dynamics with the one of the estimation error $e=$ $\tilde{z}-\tilde{x}$, the full dynamics is given by

$$
\begin{aligned}
\dot{\tilde{x}} & =(A+B F) \tilde{x}+B F e+B r \\
\dot{e} & =(A-G C) e
\end{aligned}
$$


that is the proof of the Separation Principle.

A weakness of the procedure described above has been put in evidence in [7] and arises once the solution is applied to the original nonlinear model.

In order to analyse the effects of each contribution in the whole controlled system, the use of the nominal state feedback (9) is firstly introduced. The controlled dynamics can be written as

$$
\dot{x}=f(x)+g(x)\left(F\left(x-x_{e}\right)+r\right)=F_{c}(x, r)
$$

Computing the equilibrium points, denoted as $x_{e}^{c}$ to put in evidence its origin from the controlled dynamics, one has

$$
f\left(x_{e}^{c}\right)+g\left(x_{e}^{c}\right)\left(K\left(x_{e}^{c}-x_{e}\right)+r\right)=F_{c}\left(x_{e}^{c}, r\right)=0
$$

It is easy to verify that if $r=0, x_{e}^{c}=x_{e}$. Otherwise, the new equilibrium point $x_{e}^{c}$ is different from $x_{e}$.

This change implies that, at steady state, the system is in the equilibrium point $x_{e}^{c}$.

The introduction of an observer to estimate the state for a feedback implementation must preserve this asymptotic behaviour, as it happens in the linear case, and the equilibrium point must remain $x_{e}^{c}$.

The fulfilment of this condition can be verified analysing the whole system obtained introducing the estimated state given by the observer for the state feedback (8) in the compact form (12) applied to system (1).

On the basis of the relationships between the local state $\tilde{x}$ and its estimate $\tilde{z}$, as well as between the original state $x$ and its estimate $z$, the control law (9) can be expressed, in the original coordinates, as

$$
u=F z-F x_{e}+r
$$

and the observer dynamics (11) can assume the form

$$
\dot{z}=(A-G C)\left(z-x_{e}\right)+B u+G y=(A-G C) z-(A-G C) x_{e}+B u+G y
$$

The full interconnected dynamics is then described by

$$
\begin{aligned}
& \dot{x}=f(x)+g(x) F z-g(x) F x_{e}+g(x) r \\
& \dot{z}=(A-G C+B F) z-(A+B F) x_{e}+G C x+B r
\end{aligned}
$$

In order to check the effectiveness of the controlled system (20), as a first step the error dynamics like (13) can be computed to verify its convergence to zero.

$$
\begin{aligned}
\dot{e}= & (A-G C+B F-g(x) F) e \\
& +(A+B F-g(x) F) x-f(x) \\
& -(A+B F-g(x) F) x_{e}+(B-g(x)) r
\end{aligned}
$$


Approximating (21) in a neighbourhood of $x=x_{e}$, recalling (5), one gets

$$
\dot{e}=(A-G C) e
$$

the same as in (15) as expected. That is, locally around $x_{e}$, the estimation error goes to zero. The problem is that, for the full dynamics $(20), x_{e}$ is not an equilibrium point. In fact, to compute the equilibrium points the system

$$
\begin{array}{r}
f(x)+g(x) F z-g(x) F x_{e}+g(x) r=0 \\
(A-G C+B F) z-(A+B F) x_{e}+G C x+B r=0
\end{array}
$$

must be solved. It is easy to verify that substituting $x=x_{e}$, as well as $z=x_{e}$ since the estimation error goes to zero, in (23), the condition

$$
B r=0
$$

is obtained, clearly impossible. This means that it is no more guaranteed that the dynamics evolves in a neighbourhood of $x_{e}$.

On the other hand, not even $x=x_{e}^{c}$, and then $z=x_{e}^{c}$, are equilibrium conditions for the two subsystems because, by substitution in (23), the expressions

$$
\begin{aligned}
-g\left(x_{e}^{c}\right) F x_{e} & =0 \\
(A+B F)\left(x_{e}^{c}-x_{e}\right)+B r & =0
\end{aligned}
$$

are obtained, once again impossible.

It is possible to conclude that this approach cannot work properly because $i$. the insertion of the observer dynamics interferes with the characteristics of the controlled system, changing the equilibrium point; $i i$. the observer does not work as expected, since the manifold in which the local convergence is assured does not coincide with a neighbourhood of the new equilibrium point.

In [7], an improvement in the procedure recalled above has been introduced, remaining in the locally linearised approximated context but trying to avoid the undesired effects $i$. and $i$. previously mentioned. Such a new procedure is shorty recalled in next Section 3.

\section{The Improved Design Procedure}

The idea for the solution proposed in [7] is based on the possibility of designing a state observer in such a way that the equilibrium point of the controlled system is the same both when the state is supposed to be measured and when its estimate provided by the observer is used.

Starting from the system (1)-(2), suppose it has been defined a linear state feedback control with a regulation term of the form (9), expressed in the original coordinates,

$$
u=F x+r
$$


The controlled dynamics is described by

$$
\dot{x}=f(x)+g(x) F x+g(x) r=\Phi(x, r)
$$

with output (2). Using the same notation previously adopted, be $x_{e}^{c}$ the equilibrium point for the controlled system $(27), \Phi\left(x_{e}^{c}, r\right)=0$.

The design technique is again based on a linear observer and on local convergence of the estimation error, but preserving the convergence of the system to $x_{e}^{c}$.

To this aim, the linear approximation of (27) in a neighbourhood of $x_{e}^{c}$ is computed as

$$
\dot{\bar{x}}=A_{c} \bar{x}
$$

where $A_{c}=\left.\frac{\partial \Phi(x, r)}{\partial x}\right|_{x=x_{e}^{c}}$ and $\bar{x}=x-x_{e}^{c}$. Now, a linear observer is designed on the basis of the closed loop system, i.e. an observer for the state of (27). The structure is the same as in (11), so that it has the form

$$
\dot{\bar{z}}=\left(A_{c}-G C_{c}\right) \bar{z}+G y
$$

where $y=C_{c} \bar{x}$ and $C_{c}=\left.\frac{\partial h(x)}{\partial x}\right|_{x=x_{e}^{c}}$ are defined as in the previous case for a different equilibrium point. Conditions under which the estimation error converges asymptotically to zero for the so defined problem are trivial, being $\sigma\left(A_{c}-G C_{c}\right) \in$ $C^{-}$.

The so obtained observer is used in the full closed loop system to provide a state estimation for the state feedback (26). Clearly, since $\bar{z}$ is the estimation of $\bar{x}$, that is $\lim _{t \rightarrow \infty}\|\bar{x}-\bar{z}\|=0, z=\bar{z}+x_{e}^{c}$ is the estimation of $x$; in fact $\lim _{t \rightarrow \infty}\|\bar{x}-\bar{z}\|=$ $\lim _{t \rightarrow \infty}\left\|\left(x-x_{e}^{c}\right)-\left(z-x_{e}^{c}\right)\right\|=\lim _{t \rightarrow \infty}\|x-z\|=0$

In order to study the effect of such a control scheme, the full closed loop dynamics has to be written. One has

$$
\begin{aligned}
\dot{x} & =f(x)+g(x) F z+g(x) r=\Phi(x, r)+g(x) F(z-x) \\
\dot{z} & =\left(A_{c}-G C_{c}\right) z-A_{c} x_{e}^{c}+G C_{c} x
\end{aligned}
$$

If the dynamics of the error $e=z-x$ is computed, the expression

$$
\begin{aligned}
\dot{e} & =\left(A_{c}-G C_{c}\right)(e+x)-A_{c} x_{e}^{c}+G C_{c} x-\Phi(x, r)-g(x) F e= \\
& =\left(A_{c}-G C-g(x) F\right) e+A_{c}\left(x-x_{e}^{c}\right)-\Phi(x, r)
\end{aligned}
$$

is obtained. Its approximation in a neighbourhood of $x=x_{e}^{c}$, for which

$$
\Phi(x, r)=A_{c}\left(x-x_{e}^{c}\right), \quad B_{c}=g\left(x_{e}^{c}\right)
$$

yields to

$$
\dot{e}=\left(A_{c}-G C_{c}-B_{c} F\right) e
$$


which converges, if the pair $\left(A_{c}-B_{c} F, C_{c}\right)$ is detectable, once matrix $G$ is computed to have $\sigma\left(A_{c}-B_{c} F-G C_{c}\right) \in C^{-}$.

At the same time, once the equilibrium points of (30) are computed, it is easy to verify, by straightforward substitution, that $x=x_{e}^{c}$ and $z=x_{e}^{c}$ are solutions. In fact

$$
\begin{aligned}
\Phi\left(x_{e}^{c}, r\right)+g\left(x_{e}^{c}\right) F\left(x_{e}^{c}-x_{e}^{c}\right) & =0 \\
\left(A_{c}-G C_{c}\right) x_{e}^{c}-\left(A_{c}-G C_{c}\right) x_{e}^{c} & =0
\end{aligned}
$$

Rewriting (30) in the new coordinates $(x, e)$

$$
\begin{aligned}
\dot{x} & =\Phi(x, r)+g(x) F e \\
\dot{e} & =\left(A_{c}-G C-g(x) F\right) e+A_{c}\left(x-x_{e}^{c}\right)-\Phi(x, r)
\end{aligned}
$$

and computing its linear approximation in a neighbourhood of $x=x_{e}^{c}$ as

$$
\begin{aligned}
\dot{x} & =A_{c} x+B_{c} K e-A_{c} x_{e}^{c} \\
\dot{e} & =\left(A_{c}-G C-B_{c} K\right) e
\end{aligned}
$$

the dynamical matrix

$$
A_{\text {TOT }}=\left(\begin{array}{cc}
A_{c} & B_{c} F \\
0 & \left(A_{c}-B_{c} F\right)-G C_{c}
\end{array}\right)
$$

is obtained. It shows that, even if the Separation Principle does not hold strictly, the design and the application of the linear observer after the synthesis of the state feedback control does not affect the controlled dynamics which keeps, in its linear approximation, the dynamical matrix $A_{c}$. This procedure is applied in Section 5 and the results of numerical simulations are reported in Section 6.

\section{A Different Problem Formulation}

The approach described above is a possible solution to the problem of designing a linear observer after that a state feedback with a drift term has been computed for a nonlinear dynamics, like the ones that are obtained solving a LQR problem with a reference term.

A different formulation can be adopted for the same problem, aiming at maintaining the validity of the Separation Principle and, at the same time, at taking into account the presence of the observer in the full controlled closed loop dynamics. Then, in view of the use of a linear observer for the solution of the LQR problem for a nonlinear system, the full dynamics composed by the connection between the nonlinear dynamics and the linear observer can be considered in the design problem; the feedback control law can be requested 
to be a feedback from the observer state only, and the estimation error can be considered as a further term in the cost function.

Under these hypothesis, the optimal control problem can be formulated as follows. Given a nonlinear dynamics of the form

$$
\begin{aligned}
& \dot{x}=f(x)+g(x) u \\
& \dot{z}=L z+M u+N y
\end{aligned}
$$

with $y=h(x), f\left(x_{e}\right)=0, g\left(x_{e}\right) \neq$ and $h\left(x_{e}\right)=0$, find the control $u$ which minimises the cost function

$$
J=\int_{0}^{\infty}\left(x^{T} Q x+u^{T} R u+(z-x)^{T} P(z-x)\right) d t
$$

with $P$ positive definite matrix. During the design procedure, choose properly the matrices $L, M$ and $N$.

Following the same consideration as in Section 2, if a linear approximation of the dynamics is considered, the problem can be solved in the framework of the LQR theory. Assuming the same approximation as in (4) for the first part of the dynamics and thanks to the predefined linear structure for the observer dynamics, (37) can be approximated, in a neighbourhood of $\left(x_{e}, x_{e}\right)$, as

$$
\begin{aligned}
\dot{\tilde{x}} & =A \tilde{x}+B u \\
\dot{\tilde{z}} & =L \tilde{z}+M u+N C \tilde{x}
\end{aligned}
$$

or, in a more compact form,

$$
\dot{\xi}=\hat{A} \xi+\hat{B} u
$$

where

$$
\xi=\left(\begin{array}{c}
\tilde{x} \\
\tilde{z}
\end{array}\right), \quad \hat{A}=\left(\begin{array}{cc}
A & 0 \\
N C & L
\end{array}\right), \quad \hat{B}=\left(\begin{array}{c}
B \\
M
\end{array}\right)
$$

Under these positions, also the cost function (38) can be rewritten in the compact form

$$
J=\int_{0}^{\infty}\left((\xi-d)^{T} \hat{Q}(\xi-d)+u^{T} R u\right) d t
$$

with

$$
\hat{Q}=\left(\begin{array}{cc}
Q+P & -P \\
-P & P
\end{array}\right), \quad d=-\left(\begin{array}{l}
x_{e} \\
x_{e}
\end{array}\right)
$$

It is easy to verify that the matrix $\hat{Q}$ is positive definite. The Riccati equation

$$
\hat{K} \hat{B} R^{-1} \hat{B}^{T} \hat{K}-\hat{K} \hat{A}-\hat{A}^{T} \hat{K}-\hat{Q}=0
$$

can be solved, obtaining the positive definite symmetric matrix $\hat{K}$. Following the partition induced by the structure of the matrices involved, the matrix $\hat{K}$ can be written as

$$
\hat{K}=\left(\begin{array}{ll}
K_{11} & K_{12} \\
K_{12} & K_{22}
\end{array}\right)
$$


with $K_{11}, K_{12}$ and $K_{22}$ symmetric positive definite. The block structure of the matrices involved in (44) may help to simplify the computations. Then, (44) can be written as

$$
\begin{gathered}
\left(\begin{array}{ll}
K_{11} & K_{12} \\
K_{12} & K_{22}
\end{array}\right)\left(\begin{array}{c}
B \\
M
\end{array}\right) R^{-1}\left(B^{T} M^{T}\right)\left(\begin{array}{ll}
K_{11} & K_{12} \\
K_{12} & K_{22}
\end{array}\right) \\
-\left(\begin{array}{ll}
K_{11} & K_{12} \\
K_{12} & K_{22}
\end{array}\right)\left(\begin{array}{cc}
A & 0 \\
N C & L
\end{array}\right)-\left(\begin{array}{cc}
A^{T} & C^{T} N^{T} \\
0 & L^{T}
\end{array}\right)\left(\begin{array}{ll}
K_{11} & K_{12} \\
K_{12} & K_{22}
\end{array}\right)-\left(\begin{array}{cc}
Q+P & -P \\
-P & P
\end{array}\right)=0
\end{gathered}
$$

Once the solution of the Riccati equation has been computed, the optimal control, due to the presence of the tracking term, assumes the state feedback form (as in (8))

$$
u=-R^{-1} \hat{B}^{T} \hat{K} \xi+R^{-1} \hat{B}^{T}\left(\hat{K} \hat{B} R^{-1} \hat{B}^{T}-\hat{A}^{T}\right)^{-1} \hat{Q} d=\hat{F} \xi+\hat{r}
$$

The first term is the optimal stabilising state feedback while the second one is the forcing term for the reference tracking. Focusing on the gain matrix $\hat{F}$, its structure can be put in evidence writing the matrices with their block partition. One has

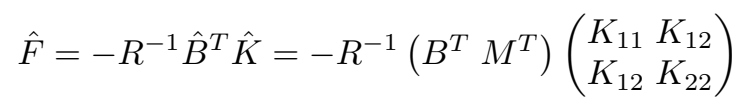

$$
\begin{aligned}
& =-R^{-1}\left(B^{T} K_{11}+M^{T} K_{12} \quad B^{T} K_{12}+M^{T} K_{22}\right)
\end{aligned}
$$

The full system (39), under the feedback control, becomes

$$
\begin{aligned}
\dot{\tilde{x}}= & \left(A-B R^{-1}\left(B^{T} K_{11}+M^{T} K_{12}\right)\right) \tilde{x}-B R^{-1}\left(B^{T} K_{12}+M^{T} K_{22}\right) \tilde{z}+B \hat{r} \\
\dot{\tilde{z}}= & \left(L-M R^{-1}\left(B^{T} K_{12}+M^{T} K_{22}\right)\right) \tilde{z} \\
& +\left(N C-M R^{-1}\left(B^{T} K_{11}+M^{T} K_{12}\right)\right) \tilde{x}+M \hat{r}
\end{aligned}
$$

Multiplying the dynamical matrix of (49) by $\left(\begin{array}{cc}I & 0 \\ -I & I\end{array}\right)$ on the left and by $\left(\begin{array}{cc}I & 0 \\ -I & I\end{array}\right)^{-1}=\left(\begin{array}{ll}I & 0 \\ I & I\end{array}\right)$ on the right, one gets the equivalent matrix

$$
\begin{gathered}
\left(\begin{array}{cc}
I & 0 \\
-I & I
\end{array}\right)\left(\begin{array}{cc}
A-B R^{-1}\left(B^{T} K_{11}+M^{T} K_{12}\right) & -B R^{-1}\left(B^{T} K_{12}+M^{T} K_{22}\right) \\
N C-M R^{-1}\left(B^{T} K_{11}+M^{T} K_{12}\right) & L-M R^{-1}\left(B^{T} K_{12}+M^{T} K_{22}\right)
\end{array}\right)\left(\begin{array}{ll}
I & 0 \\
I & I
\end{array}\right)= \\
\left(\begin{array}{cc}
A-B R^{-1}\left(B^{T}\left(K_{11}+K_{12}\right)+M^{T}\left(K_{12}+K_{22}\right)\right) & -B R^{-1}\left(B^{T} K_{12}+M^{T} K_{22}\right) \\
L+N C-A-(M-B) R^{-1}\left(B^{T}\left(K_{11}+K_{12}\right)+M^{T}\left(K_{12}+K_{22}\right)\right) & L-(M-B) R^{-1}\left(B^{T} K_{12}+M^{T} K_{22}\right)
\end{array}\right)
\end{gathered}
$$

On the basis of these expressions, two different hypothesis can be formulated in order to have the feedback control depending on the observer state only. 


\subsection{First Hypothesis}

According to the classical observer based control schemes, it can be introduced the constraint that the control law is a feedback from the estimated state $\tilde{z}$ only; this means that in (48) it must be verified that

$$
B^{T} K_{11}+M^{T} K_{12}=0
$$

It is interesting to notice that this position makes the observer dynamics independent from the not measurable state $\tilde{x}$, since the term $N C \tilde{x}=N y$ is measurable.

Under condition (51), the control law is

$$
u=-B R^{-1}\left(B^{T} K_{12}+M^{T} K_{22}\right) \tilde{z}+B \hat{r}
$$

where the forcing term $\hat{r}$, written putting in evidences the block decomposition of the matrices involved, assumes the expression

$$
\begin{gathered}
\hat{r}=-R^{-1}\left(B^{T} M^{T}\right) \times \\
\left(\begin{array}{c}
-C^{T} N^{T} \\
-A^{T} \\
\left.\left(K_{12} B+K_{22} M\right) R^{-1} B^{T}\left(K_{12} B+K_{22} M\right) R^{-1} M^{T}-L^{T}\right)^{-1}\left(\begin{array}{c}
Q x_{e} \\
0
\end{array}\right)
\end{array}\right.
\end{gathered}
$$

In (53), it should be verified, as it will be done later, that the matrix

$$
\left(\begin{array}{c}
-A^{T} \\
\left(K_{12} B+K_{22} M\right) R^{-1} B^{T}\left(K_{12} B+K_{22} M\right) R^{-1} M^{T}-L^{T}
\end{array}\right)
$$

is still full rank, after the design procedure, even with the introduction of the constraint (51). If (51) holds, Riccati equation (46) is rewritten as

$$
\begin{aligned}
& \left(\begin{array}{cc}
0 & 0 \\
0 & \left(K_{12} B+K_{22} M\right) R^{-1}\left(B^{T} K_{12}+M^{T} K_{22}\right)
\end{array}\right)-\left(\begin{array}{ll}
K_{11} A+K_{12} N C & K_{12} L \\
K_{12} A+K_{22} N C & K_{22} L
\end{array}\right) \\
& -\left(\begin{array}{cc}
A^{T} K_{11}+C^{T} N^{T} K_{12} & A^{T} K_{12}+C^{T} N^{T} K_{22} \\
L^{T} K_{12} & L^{T} K_{22}
\end{array}\right)-\left(\begin{array}{cc}
Q+P & -P \\
-P & P
\end{array}\right)=0
\end{aligned}
$$

From (55), four equations can be obtained

$$
\begin{gathered}
-\left(K_{11} A+K_{12} N C\right)-\left(A^{T} K_{11}+C^{T} N^{T} K_{12}\right)-(Q+P)=0 \\
-K_{12} L-\left(A^{T} K_{12}+C^{T} N^{T} K_{22}\right)+P=0 \\
-\left(K_{12} A+K_{22} N C\right)-L^{T} K_{12}+P=0 \\
\left(K_{12} B+K_{22} M\right) R^{-1}\left(B^{T} K_{12}+M^{T} K_{22}\right)-K_{22} L-L^{T} K_{22}-P=0
\end{gathered}
$$

The sum of the four equations gives

$$
\begin{aligned}
& \left(K_{12} B+K_{22} M\right) R^{-1}\left(B^{T} K_{12}+M^{T} K_{22}\right) \\
& -\left(K_{12}+K_{22}\right)(L+N C)-(L+N C)^{T}\left(K_{12}+K_{22}\right) \\
& -\left(K_{11}+K_{12}\right) A-A^{T}\left(K_{11}+K_{12}\right)-Q=0
\end{aligned}
$$


An analysis of (60), in which both the term $K_{12} B+K_{22} M$ and the term $K_{12}+K_{22}$ are present, suggests that if the position $M=B$ is set, the whole expression (60) simplifies. On the other hand, $M=B$ means that in the observer design, the same effect of the input as in the original dynamics is chosen, so that in the observation error the contribution of the input is neglected. It corresponds to the choice of a Luenberger classic observer. Moreover, if $M=B$ is used in the expression of the matrix (50), in addition to (51), one gets

$$
\left(\begin{array}{cc}
A-B R^{-1} B^{T}\left(K_{12}+K_{22}\right) & -B R^{-1} B^{T}\left(K_{12}+K_{22}\right) \\
L+N C-A & L
\end{array}\right)
$$

With $M=B$, constraint (51) yields to

$$
K_{11}+K_{12}=0 \leftrightarrow K_{12}=-K_{11}
$$

and the four equations (56)-(59) simplify as

$$
\begin{gathered}
-K_{11}(A-N C)-(A-N C)^{T} K_{11}-(Q+P)=0 \\
K_{11} L+\left(A^{T} K_{11}-C^{T} N^{T} K_{22}\right)+P=0 \\
\left(K_{11} A-K_{22} N C\right)+L^{T} K_{11}+P=0 \\
\left(-K_{11}+K_{22}\right) B R^{-1} B^{T}\left(-K_{11}+K_{22}\right)-K_{22} L-L^{T} K_{22}-P=0
\end{gathered}
$$

and (60) becomes

$$
\begin{aligned}
& \left(-K_{11}+K_{22}\right) B R^{-1} B^{T}\left(-K_{11}+K_{22}\right) \\
& -\left(-K_{11}+K_{22}\right)(L+N C)-(L+N C)^{T}\left(-K_{11}+K_{22}\right)-Q=0
\end{aligned}
$$

Setting $L+N C=A$, equation (67) corresponds to the Riccati equation which arises when the LQR problem is defined for the system (4), the plant part of (37), and with cost function (6). In addition, it must be observed that the gain matrix in (52) reduces to $-R^{-1} B^{T}\left(-K_{11}+K_{22}\right)$, that is the same obtained solving (67). Then, the relation $L+N C=A$ is defined and the LQR problem with full state measurement for (4) and (6) can be firstly solved, finding the positive definite symmetric matrix $K_{s}=-K_{11}+K_{22}$ and using it in the full problem.

Moreover, under all these hypothesis, matrix (61) becomes

$$
\left(\begin{array}{cc}
A-B R^{-1} B^{T}\left(-K_{11}+K_{22}\right) & -B R^{-1} B^{T}\left(-K_{11}+K_{22}\right) \\
0 & A-N C
\end{array}\right)
$$

from which it can be concluded that the closed loop system (49) is asymptotically stable if $A-N C$ has all its eigenvalues with negative real part, since the block $A-B R^{-1} B^{T}\left(-K_{11}+K_{22}\right)$ is asymptotically stable from the reduced LQR problem solution. 
Finally, it is possible to rewrite the optimal control law (47) in a simpler form, particularising (52) and (53). One gets

$$
\begin{aligned}
u= & -R^{-1} B^{T} K_{s} \tilde{z} \\
& -R^{-1} B^{T}\left(\begin{array}{ll}
I & I
\end{array}\right)\left(\begin{array}{cc}
-A^{T} & -(N C)^{T} \\
K_{s} B R^{-1} B^{T} & K_{s} B R^{-1} B^{T}-L^{T}
\end{array}\right)^{-1}\left(\begin{array}{c}
Q x_{e} \\
0
\end{array}\right)
\end{aligned}
$$

Since

$$
\begin{gathered}
\left(\begin{array}{cc}
-A^{T} & -(N C)^{T} \\
K_{s} B R^{-1} B^{T} & K_{s} B R^{-1} B^{T}-L^{T}
\end{array}\right)= \\
=\left(\begin{array}{cc}
I & -I \\
0 & I
\end{array}\right)\left(\begin{array}{cc}
-A^{T}+K_{s} B R^{-1} B^{T} & 0 \\
K_{s} B R^{-1} B^{T} & -L^{T}
\end{array}\right)\left(\begin{array}{ll}
I & I \\
0 & I
\end{array}\right)
\end{gathered}
$$

computing the inverse of both members, one has that

$$
\begin{gathered}
\left(\begin{array}{cc}
-A^{T} & -(N C)^{T} \\
K_{s} B R^{-1} B^{T} & K_{s} B R^{-1} B^{T}-L^{T}
\end{array}\right)^{-1}= \\
=\left(\begin{array}{cc}
I & -I \\
0 & I
\end{array}\right)\left(\begin{array}{cc}
-\left(A^{T}-K_{s} B R^{-1} B^{T}\right)^{-1} & 0 \\
-\left(L^{T}\right)^{-1}\left(K_{s} B R^{-1} B^{T}\right)\left(A^{T}-K_{s} B R^{-1} B^{T}\right)^{-1} & -\left(L^{T}\right)^{-1}
\end{array}\right)\left(\begin{array}{ll}
I & I \\
0 & I
\end{array}\right)
\end{gathered}
$$

which can be computed since both $A^{T}-K_{s} B R^{-1} B^{T}$ and $L$ are invertible by construction.

With all these positions, the expression for the optimal control (69) becomes

$$
\begin{aligned}
u= & -R^{-1} B^{T} K_{s} \tilde{z} \\
& -R^{-1} B^{T}\left(\begin{array}{ll}
I & 0
\end{array}\right)\left(\begin{array}{cc}
-\left(A^{T}-K_{s} B R^{-1} B^{T}\right)^{-1} & 0 \\
* & -\left(L^{T}\right)^{-1}
\end{array}\right)\left(\begin{array}{c}
Q x_{e} \\
0
\end{array}\right)= \\
= & -R^{-1} B^{T} K_{s} \tilde{z}+R^{-1} B^{T}\left(A^{T}-K_{s} B R^{-1} B^{T}\right)^{-1} Q x_{e}
\end{aligned}
$$

Comparing the expression (8) for the control when the state feedback without observer is considered (obtained in Section 2), with (74) obtained with the proposed procedure, it clear that (74) corresponds to (8) with the state $\tilde{x}$ substituted by the estimated state $\tilde{z}$.

\subsection{Second Hypothesis}

A different choice can be performed, instead of introducing the constraint (51) to have the feedback control law depending on the observer state only. It can be assumed that, once the control (47) is computed, the state $\tilde{x}$ is substituted by its estimate $\tilde{z}$. With this position, dynamics (49) becomes

$$
\begin{aligned}
\dot{\tilde{x}} & =A \tilde{x}-B R^{-1}\left(B^{T} K_{11}+M^{T} K_{12}+B^{T} K_{12}+M^{T} K_{22}\right) \tilde{z}+B \hat{r} \\
\dot{\tilde{z}} & =\left(L-M R^{-1}\left(B^{T} K_{12}+M^{T} K_{22}+B^{T} K_{11}+M^{T} K_{12}\right)\right) \tilde{z}+N C \tilde{x}+M \hat{r}
\end{aligned}
$$


while matrix (50) assumes the expression

$$
\left(\begin{array}{ll}
m_{11} & m_{12} \\
m_{21} & m_{22}
\end{array}\right)
$$

with

$$
\begin{aligned}
& m_{11}=A-B R^{-1}\left(B^{T}\left(K_{11}+K_{12}\right)+M^{T}\left(K_{12}+K_{22}\right)\right) \\
& m_{12}=-B R^{-1}\left(B^{T}\left(K_{11}+K_{12}\right)+M^{T}\left(K_{12}+K_{22}\right)\right) \\
& m_{21}=L+N C-A-(M-B) R^{-1}\left(B^{T}\left(K_{11}+K_{12}\right)+M^{T}\left(K_{12}+K_{22}\right)\right) \\
& m_{22}=L-(M-B) R^{-1}\left(B^{T}\left(K_{11}+K_{12}\right)+M^{T}\left(K_{12}+K_{22}\right)\right)
\end{aligned}
$$

It can be verified that, setting $M=B$ and $L=A-N C$, matrix (76) reduces to

$$
\left(\begin{array}{c}
A-B R^{-1} B^{T}\left(K_{11}+2 K_{12}+K_{22}\right)-B R^{-1} B^{T}\left(K_{11}+2 K_{12}+K_{22}\right) \\
0
\end{array}\right)
$$

At the same time, if the Riccati equation (46) is multiplied on the left by (I I) and on the right by $\left(\begin{array}{l}I \\ I\end{array}\right)$, using the relations defined above for $M$ and $L$, easy computations give the expression

$$
\begin{gathered}
\left(K_{11}+2 K_{12}+K_{22}\right) B R^{-1} B^{T}\left(K_{11}+2 K_{12}+K_{22}\right) \\
-\left(K_{11}+2 K_{12}+K_{22}\right) A-A^{T}\left(K_{11}+2 K_{12}+K_{22}\right)-Q=0
\end{gathered}
$$

Defining, in this case,

$$
K_{s}=K_{11}+2 K_{12}+K_{22}
$$

matrix (77) becomes the same as (68), which results asymptotically stable since $K_{s}$ is the solution of the Riccati equation (78) and $N$ is chosen so that $A-N C$ is asymptotically stable, provided that $(A, C)$ is observable.

Both the hypothesis adopted in Subsection 4.1 and in the present Subsection 4.2 bring to the same procedure for the control system design. It consists in solving the LQR problem defined by dynamics (4) under cost function (6), solving a Riccati equation of the form (7), with $K=K_{s}$, and using the state feedback (12) using the estimate state $\tilde{z}$ provided by the linear observer (11) with $G=N$ computed to have $A-N C$ asymptotically stable.

The behaviour and the performances of this approach are compared with the same of the approach described in Section 3 making use of the numerical example introduced in next Section.

\section{The Case Study}

As anticipated in the Introduction, the case study of an epidemic spread is considered for comparing the proposed approaches. The mathematical model, briefly 
recalled in Subsection 5.1, is the one introduced in [3] and [4], for which a LQR optimal control has been proposed in [5]. The classical state feedback solution there obtained has been enhanced introducing a state observer in [6], whose design is here shortly recalled in Subsection 5.2, necessary since (at least) one of the state variable is not measurable. Finally, in [7], an improvement in the observer design is introduced to compensate some of the effects of the nonlinearity of the dynamics, as recalled in Subsection 5.4. The solution here proposed, described in Section 4, is applied in Subsection 5.5.

\subsection{A Short Recall of the Mathematical Model}

In this paper, the model of the HIV/AIDS diffusion presented in $[3,4]$ is adopted and is here briefly recalled.

The state variables introduced in the model denote the healthy people $S_{1}$, not aware of dangerous behaviours and then can be infected, and $S_{2}$, the ones that, suitably informed, give great attention to the protection, and the three levels of infectious subjects: $I$, the infected but unaware of their status, $P$, the HIV positive patients, $A$, the AIDS diagnosed ones.

The control actions are the information campaign, $u_{1}$ and the test campaign to discover the infection as soon as possible, $u_{2}$. A third action, $u_{3}$, the therapy which aims at reducing the transition from HIV to AIDS, is also considered, since mortality among $A$ is higher that in $P$. Then, the mathematical model is

$$
\begin{aligned}
\dot{S}_{1} & =Z-d S_{1}-\beta \frac{S_{1} I}{N_{c}}+\gamma S_{2}-S_{1} u_{1} \\
\dot{S}_{2} & =-(\gamma+d) S_{2}+S_{1} u_{1} \\
\dot{I} & =\beta \frac{S_{1} I}{N_{c}}-(d+\delta) I-\psi \frac{I}{N_{c}} u_{2} \\
\dot{P} & =\varepsilon \delta I-(\alpha+d) P+\phi \psi \frac{I}{N_{c}} u_{2}+P u_{3} \\
\dot{A} & =(1-\varepsilon) \delta I+\alpha P-(\mu+d) A+(1-\phi) \psi \frac{I}{N_{c}} u_{2}-P u_{3}
\end{aligned}
$$

where $N_{c}=S_{1}+S_{2}+I$. In (80), $d$ denotes the rate of natural death; $Z$ denotes the flux of new subjects in the class $S_{1} ; \beta$ is related to the dangerous interactions between $S_{1}$ and $I$ categories; $\gamma$ is the rate of wise subjects that could change, incidentally, their status, increasing $S_{1}(t) ; \psi$ is related to the control action aiming at helping the individuals in class $I$ to discover their infectious condition, and therefore to flow to the $P$ or the $A$ class; $\phi$ is the percentage of test positive subjects with HIV $((1-\phi)$ the percentage with AIDS); $\delta$ is the rate of transition from $I$ to $P$ (percentage $\varepsilon$ ) or $A$ (percentage $(1-\varepsilon)$ ) without any external action; $\alpha$ is the rate of the natural transition from $P$ to $A ; \mu$ is the rate of death in class $A$ caused by the infection.

The compact form for (80) can be expressed as

$$
\dot{X}=f(X)+g(X) U=F(X, U)
$$


once $X=\left(\begin{array}{lllll}S_{1} & S_{2} & I & P & A\end{array}\right)^{T}, U=\left(\begin{array}{lll}u_{1} & u_{2} & u_{3}\end{array}\right)^{T}$ and

$$
\begin{gathered}
f(X)=\left(\begin{array}{c}
Z-d S_{1}-\frac{\beta S_{1} I}{N_{c}}+\gamma S_{2} \\
-(\gamma+d) S_{2} \\
\frac{\beta S_{1} I}{N_{c}}-(d+\delta) I \\
\varepsilon \delta I-(\alpha+d) P \\
(1-\varepsilon) \delta I+\alpha P-(\mu+d) A
\end{array}\right) \\
g(X)=\left(\begin{array}{llll}
g_{1} & g_{2} & g_{3}
\end{array}\right)=\left(\begin{array}{ccc}
-S_{1} & 0 & 0 \\
S_{1} & 0 & 0 \\
0 & -\psi \frac{I}{N_{c}} & 0 \\
0 & \phi \psi \frac{I}{N_{c}} & P \\
0 & (1-\phi) \psi \frac{I}{N_{c}} & -P
\end{array}\right)
\end{gathered}
$$

are defined.

For the choice of possible output functions, it must be observed that the subject with a positive diagnosis, $P$ and $A$, can be easily measured, since reported by medical operators.

Then, it seems reasonable to assume the measure of the total number of diagnosed individuals, $P(t)+A(t)$, as the possible meaningful output, so giving

$$
y(t)=C X(t), \quad C=\left(\begin{array}{lllll}
0 & 0 & 0 & 1 & 1
\end{array}\right)
$$

\subsection{Control Problem Definiton and LQR Problem Formulation}

An optimal control problem for the HIV/AIDS dynamics (80) has been formulated in [5]. It aims at minimising the most dangerous class of individuals, the infected $I$, keeping the control amplitude as low as possible. With these choices, the distinction between $P$ and $A$, as well as the presence of the control $u_{3}$, become not relevant. Then, the two-dimensional control vector $\hat{U}=\left(u_{1} u_{2}\right)^{T}$ is introduced, neglecting, consequently, the vector field $g_{3}(\cdot)$ in (83) introducing the matrix $\hat{g}(X)=\left(g_{1} g_{2}\right)$.

Under these positions, the cost function

$$
J(X, \hat{U})=\frac{1}{2} \int_{t_{0}}^{\infty}\left(q I^{2}+r_{1} u_{1}^{2}+r_{2} u_{2}^{2}\right) d t=\frac{1}{2} \int_{t_{0}}^{\infty}\left(X^{T} Q X+\hat{U}^{T} R \hat{U}\right) d t
$$

is defined, with $Q$ the five dimensional square matrix with all zero entries except $Q(3,3)=q$, and $R=\left(\begin{array}{cc}r_{1} & 0 \\ 0 & r_{2}\end{array}\right), r_{1}, r_{2}>0$.

The quadratic structure of (85) and the preference for a state feedback implementability of the control law drove the solution of such problem in [5] to a LQR form designed on the linearised approximation of (80) in the neighbourhood of one equilibrium point. A study of the existence of equilibrium points and of their stability properties has been performed in $[3,4]$, yielding to the two 
possible solutions

$$
X_{1}^{e}=\left(\begin{array}{c}
1 / d \\
0 \\
0 \\
0 \\
0
\end{array}\right) Z \quad X_{2}^{e}=\left(\begin{array}{c}
1 / H \\
0 \\
\frac{H-d}{H(d+\delta)} \\
\frac{\varepsilon \delta(H-d)}{H(\alpha+d)(d+\delta)} \\
\frac{\delta(H-d)[(1-\varepsilon) d+\alpha]}{H(\alpha+d)(d+\delta)(\mu+d)}
\end{array}\right) Z
$$

where $H=\beta-\delta$. The non negativeness of the elements in the vector state $X_{2}^{e}$ implies the condition $H \geq d>0$; therefore the equilibrium point $X_{2}^{e}$ is a feasible one if and only if $H \geq d$, being $X_{1}^{e}=X_{2}^{e}$ if $H=d$. The presence of a bifurcation in the stability analysis is discussed in [4].

Making use of the same values for the model parameters as in [5], condition $H>d$ holds, so that both the equilibrium points exist. Computing the two linearised dynamics of (81) in the neighbourhood of the two equilibrium points one gets

$$
\begin{aligned}
\dot{\tilde{X}} & =A_{i} \tilde{X}+\hat{B}_{i} \hat{U} \\
\tilde{y} & =C \tilde{X}
\end{aligned}
$$

with $A_{i}=\left.\frac{\partial f}{\partial X}\right|_{X=X_{i}^{e}}, \hat{B}_{i}=\hat{g}\left(X_{i}^{e}\right) \tilde{y}=C \tilde{X}=y-C X_{i}^{e}$ and

$$
\tilde{X}=X-X_{i}^{e}
$$

$i=1,2$ depending on the choice.

Despite the procedure can be adopted making reference to both the equilibrium points, easy computations show that the linear dynamics which approximates the nonlinear one in the neighbourhood of $X_{1}^{e}$ is neither detectable nor controllable. So, in view of a control synthesis in the local linear domain, the linearisation in a neighbourhood of $X_{2}^{e}$ is chosen. Then, the linear dynamics is (87) with $i=2$.

\subsection{The Linearised Optimal Control Problem Solution}

The use of the new coordinates $\tilde{X}$, consequence of (88), implies a change of variables also in the cost function (85), where $\tilde{X}$ must appear instead of $X$. The new expression is

$$
\begin{aligned}
& J(X, \hat{U})=J\left(\tilde{X}+X_{2}^{e}, \hat{U}\right)=\tilde{J}(\tilde{X}, \hat{U})= \\
& =\frac{1}{2} \int_{t_{0}}^{\infty}\left(\left(\tilde{X}^{T}-\bar{r}\right) Q(\tilde{X}-\bar{r})+\hat{U}^{T} R \hat{U}\right) d t \\
& =\frac{1}{2} \int_{t_{0}}^{\infty}\left(q\left(\tilde{I}(t)+\frac{H-d}{H(d+\delta)}\right)^{2}+r_{1} u_{1}^{2}(t)+r_{2} u_{2}^{2}(t)\right) d t
\end{aligned}
$$

where $\bar{r}=\left(* * \bar{r}_{\tilde{I}} * *\right)^{T}$ denotes the LQR tracking term, with $\bar{r}_{\tilde{I}}=-\frac{H-d}{H(d+\delta)}$. 
For a dynamics (87), the optimal control problem with cost function (89) corresponds to a classical Linear Quadratic Regulator (LQR) problem with a constant tracking term. The result, computed and discussed in [5], is a state feedback control law with a reference term. Once the Algebraic Riccati Equation

$$
0=K_{R} \hat{B}_{2} R^{-1} \hat{B}_{2}^{T} K_{R}-K_{R} A_{2}-A_{2}^{T} K_{R}-Q
$$

is solved w.r.t. $K_{R}$, the state feedback optimal control law is given by ([1])

$$
\hat{U}=-R^{-1} \hat{B}_{2}^{T} K_{R} \tilde{X}+R^{-1} \hat{B}_{2}^{T} g_{\bar{r}}=F \tilde{X}+r
$$

where $g_{\bar{r}}=\left(K_{R} \hat{B}_{2} R^{-1} B_{2}^{T}-A_{2}^{T}\right)^{-1} Q \bar{r}$ with $Q \bar{r}=\left(\begin{array}{lllll}0 & 0 & q_{\bar{r}_{\tilde{I}}} & 0 & 0\end{array}\right)^{T} ; K=-R^{-1} \hat{B}_{2}^{T} K_{R}$ is the gain matrix as in (9) while $r=R^{-1} \hat{B}_{2}^{T} g_{\bar{r}}$ is the constant tracking term $r$.

Stability for the linear controlled system is proven in [5]. In the same paper, the problem of the unavailability of a measure of all the state variables has been solved computing a linear state observer under the hypothesis that, once only local solutions are available, due to the request of a state feedback control, then also for the observer a linear approach can be sufficient, whose approximation is well compensated by its simplicity of design and implementation.

Then, once verified the detectability property on $\left(A_{2}, C\right)$ in $(87)(i=2)$, the state estimation $\tilde{z}(t)$ of the state $\tilde{X}$ verifying the asymptotic condition $\lim _{t \rightarrow+\infty}\|\tilde{X}(t)-\tilde{z}(t)\|=0$ can be obtained as the state evolution of the Luenberger like linear observer

$$
\dot{\tilde{z}}(t)=\left(A_{2}-G C\right) \tilde{z}(t)+\hat{B}_{2} \hat{U}(t)+G \tilde{y}(t)
$$

with matrix $G$ chosen in order to have all the eigenvalues of the dynamic matrix $\left(A_{2}-G C\right)$ with negative real part. The whole control system is a dynamical output feedback control with state observer and feedback from the state estimation.

\subsection{The Solution With The Improved Observer Design}

The approach proposed in [7] is here recalled. Following what illustrated in Section 3, consider the control law of the form (91) computed solving the LQR control problem on the basis of the linear approximation of the dynamics in a neighbourhood of $X_{2}^{e}$. Under the action of this state feedback, the controlled system assumes the form

$$
\dot{X}=f(X)+\hat{g}(X)(F \tilde{X}+r)=\Phi(X, r)
$$

with its linear approximation asymptotically stable. Its equilibrium point can be denoted by $X_{e}^{c}: \Phi\left(X_{e}^{c}, r\right)=0$ and the linear approximation of (93) in the neighbourhood of $X_{e}^{c}$ can be computed; it is given by

$$
\dot{\bar{X}}=A_{c} \bar{X}
$$


where $A_{c}=\left.\frac{\partial \Phi(X, r)}{\partial X}\right|_{X=X_{e}^{c}}$ and $\bar{X}=X-X_{e}^{c}$.

A local linear observer for the linear approximating dynamics can be designed in the usual form

$$
\dot{\bar{z}}=\left(A_{c}-G C\right) \bar{z}+G \bar{y}
$$

when dealing directly with the linearised dynamics, or

$$
\dot{z}=\left(A_{c}-G C\right) z+G y-A_{c} X_{e}^{c}
$$

expressed in the original state variables. According to the general discussion in Section 3, once the observer has been designed, the control law (91) can be implemented using the state estimate $\bar{z}$ instead of the real but not measurable state $\bar{X}$ (or $z$ instead of $X$ ). Then, the control law (91) can be rewritten as

$$
\begin{aligned}
u & =F \tilde{X}+r=F\left(X-X_{2}^{e}\right)+r=F\left(\bar{X}+X_{e}^{c}-X_{2}^{e}\right)+r= \\
& =F \bar{X}+r+F\left(X_{e}^{c}-X_{2}^{e}\right)
\end{aligned}
$$

so that the dynamics (93), using the observer (95), assumes the expression

$$
\dot{X}=f(X)+\hat{g}(X)(F \bar{z}+r)+\hat{g}(X) F\left(X_{e}^{c}-X_{2}^{e}\right)
$$

to be considered along with the observer dynamics (95). Some manipulations allow to write the dynamics (98) as

$$
\begin{aligned}
\dot{X} & =f(X)+\hat{g}(X)(F \bar{z}+r)+\hat{g}(X) F\left(X_{e}^{c}-X_{2}^{e}\right)= \\
& =\Phi(X, r)+\hat{g}(X) F\left(\bar{z}-\tilde{X}+X_{e}^{c}-X_{2}^{e}\right)=\Phi(X, r)+\hat{g}(X) F(z-X)
\end{aligned}
$$

where $z-X$ can be replaced by $\bar{z}-\bar{X}$ or $\tilde{z}-\tilde{X}$ according to the convenience. It is easy to verify by substitution that the whole dynamics (95)-(99) has the equilibrium point $X=X_{e}^{c}, z=X_{e}^{c}(\bar{X}=\bar{z}=0)$. This means that asymptotically the state of the observer and one of the original system are equal. The fact that the asymptotic error is equal to zero can be proved also computing the error dynamics

$$
\dot{e}=\left(A_{c}-G C\right) \bar{z}+G C \bar{x}-F(X, r)-\hat{g}(X) K(z-x)
$$

and evaluating it in a neighbourhood of $X=X_{e}^{c}$, yielding

$$
\begin{aligned}
\dot{e} & =\left(A_{c}-G C\right) \bar{z}+G C \bar{x}-A_{c} \bar{x}-\hat{B}_{c} K(\bar{z}-\bar{x})= \\
& ==\left(A_{c}-G C+\hat{B}_{c} K\right) e
\end{aligned}
$$

Then, with $\sigma\left(A_{c}-G C+\hat{B}_{c} K\right) \in C^{-}$the error goes asymptotically to zero.

It is confirmed what stated in the previous Section: the observer (95) works properly, without producing undesired changes in the system dynamics and converging asymptotically to the system state, once $G$ is designed to have $\sigma\left(A_{c}-\right.$ $\left.G C-\hat{B}_{c} K\right) \in C^{-}$, provided that $\left(A_{c}-\hat{B}_{c} K, C\right)$ is a detectable pair. 


\subsection{The Proposed Combined Approach}

Following the description reported in Section 4, the solution of the problem consists of the two steps. The first one concerns the design of the optimal state feedback control computing matrix $K_{s}$ as the solution of the Riccati equation (67) which, for the present example, assumes the expression (90) with $K_{R}=K_{s}$, $G=N$, and where $A_{2}=L-N C$. The solution (91) corresponds to the expression (74). With respect to the initial formulation of the problem in Section $4, K_{s}$ can represent $-K_{11}+K_{22}$ if the choice described in Subsection 4.1 is adopted, or $K_{11}+2 K_{12}+K_{22}$ for the choice of Subsection 4.2.

The second step is the observer design, for which $N$ and $L=A_{2}-N C$ must be computed. Under the observability condition on $\left(A_{2}, C\right), N$ can be computed so that $n$ eigenvalues with negative real part can be set to matrix $L$.

\section{Numerical Results and Discussion}

In this Section, a numerical analysis is performed to compare the solution proposed in [7] and the one obtained following the approach her proposed in Section 4 . The values for the parameters in the dynamics (80) adopted for the numerical computations have been taken from $[5,11,3,10]$ :

$$
\begin{array}{cccc}
d=0.02, & \beta=1.5, \quad \delta=0.4, & \varepsilon=0.6, \quad \phi=0.95 \\
\gamma=0.2 & \psi=10^{5}, \quad \alpha=0.5, & \mu=1, & Z=1000
\end{array}
$$

Then, $H=\beta-\delta=1.1>0$ so that the equilibrium point $X_{2}^{e}$ exists and it is locally asymptotically stable. Numerically, $X_{2}^{e}=\left(\begin{array}{lllll}0.91 & 0 & 2.34 & 1.08 & 0.9\end{array}\right)^{T} \cdot 10^{3}$.

The linear approximation in the neighbourhood of this equilibrium point is described by $C$ as in (84) and by the following numerical matrices:

$$
\begin{gathered}
A_{2}=\left(\begin{array}{ccccc}
-0.80 & 0.20 & -0.12 & 0 & 0 \\
0 & -0.22 & 0 & 0 & 0 \\
0.78 & 0 & 0.30 & 0 & 0 \\
0 & 0 & 0.24 & -0.52 & 0 \\
0 & 0 & 0.16 & 0.5 & -1.02
\end{array}\right) \\
\hat{B}_{2}=\left(\begin{array}{cc}
-0.91 & 0 \\
0.91 & 0 \\
0 & -72 \\
0 & 68.40 \\
0 & 3.60
\end{array}\right) 10^{3}
\end{gathered}
$$

\subsection{Case of Improved Observed Design}

As far as the controller is concerned, the control law is computed as the solution of the LQR problem with offset (tracking) term defined in Subsection 5.3 thanks to the controllability property of $A_{2}, \hat{B}_{2}$ in (102) and (103). Performing the 
computations, the LQR reference term $\bar{r}_{\tilde{I}}$ to be used in (91) assumes the value $\bar{r}_{\tilde{I}}=-2.34 \cdot 10^{3}$.

As in [5], the cost function weights $q=10^{-4}, r_{1}=1, r_{2}=1000$ are chosen. The solution $K_{R}$ of the Algebraic Riccati Equation (90) gives

$$
K_{R}=\left(\begin{array}{ccccc}
0.07 & -0.01 & 0.14 & 0 & 0 \\
-0.01 & 0.02 & -0.05 & 0 & 0 \\
0.14 & -0.05 & 4.33 & 0 & 0 \\
0 & 0 & 0 & 0 & 0 \\
0 & 0 & 0 & 0 & 0
\end{array}\right) 10^{-6}
$$

and then $g_{\bar{r}}=(0.050-1.0300)^{T} \cdot 10^{-2}$.

The optimal control (91) so obtained, which should drive the state variable $\tilde{I}$ of the linearised system to the reference value $\bar{r}_{\tilde{I}}$, is of the form (91) with

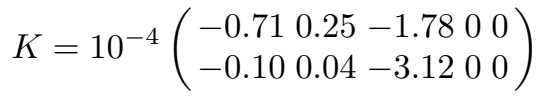

and

$$
r=\left(\begin{array}{l}
0.41 \\
0.74
\end{array}\right)
$$

As far as the observer design is concerned, following the procedure described in Section 3, the observer to be designed has the form (29) rewritten as in (30) and here reported for the present case

$$
\dot{z}=\left(A_{c}-G C\right) z+G C x-A_{c} X_{e}^{c}
$$

where $G$ has to be computed, according to (33), after having verified the detectability property for the pair $\left(A_{c}-B_{c} K, C\right)$, in order to have $\sigma\left(A_{c}-B_{c} K-\right.$ $G C) \in C^{-}$.

The numerical value of the equilibrium point $X_{e}^{c}$ for the controlled system (93) is

$$
X_{e}^{c}=10^{4}(1.01763 .9822000)^{T}
$$

For the computation of the matrix $A_{c}-B_{c} K_{R}, A_{c}$ has to be computed as the Jacobian of the controlled system evaluated in (108), $B_{c}=g\left(X_{e}^{c}\right)$, while $K_{R}$ is the output of the LQR optimal control problem previously solved. One has

$$
A_{c}=\left(\begin{array}{ccccc}
1.09 & 0.21 & 1.04 & 0 & 0 \\
-1.11 & -0.23 & -1.34 & 0 & 0 \\
0 & 0 & -0.34 & 0 & 0 \\
0 & 0 & 0.24 & -0.52 & 0 \\
0 & 0 & 0.16 & 0.50 & -1.02
\end{array}\right)
$$

and, then,

$$
A_{c}+B_{c} K=\left(\begin{array}{ccccc}
1.04 & 0.21 & 0.92 & 0 & 0 \\
-1.06 & -0.23 & -1.22 & 0 & 0 \\
-0.76 & -0.01 & -23.40 & 0 & 0 \\
0.72 & 0.01 & 22.15 & -0.52 & 0 \\
0.04 & 0 & 1.31 & 0.50 & -1.02
\end{array}\right)
$$


Observability of the couple $\left.A_{c}-B_{c} K_{R}, C\right)$ can easily be checked and then it is possible to compute $G$ so to verify the convergence condition. Discussion about the characteristics of the transient in the observer dynamics are reported in [5] and they bring to the choice of the set of eigenvalues $\Lambda=$ $\{-1.0,-1.1,-1.2,-1.3,-1.4\}$ to be assigned to the matrix $A_{c}-B_{c} K_{R}+G C$. One has

$$
G=10^{3}(-0.221 .200 .03-0.020)^{T}
$$

\subsection{Case of the Solution Proposed}

The control law design for this case follows the same steps as for the previous case. Firstly the solution of the Riccati equation (90) with $A_{2}$ as in (102), $\hat{B}_{2}$ as in (103) and where $K_{R}=K_{s}$ has to be computed. With the same choice of the cost function weights $q=10^{-4}, r_{1}=1, r_{2}=1000$, the solution $K_{s}$ of (90) is the same as $K_{R}$ in (104). As a consequence, the same holds for $g_{\bar{r}}$.

Since the solution here adopted is based on the use of a linear state observer of the form as in (39) with $M=\hat{B}_{2}, L=A_{2}-N C$ and $N$ computed in order to have $\operatorname{sigma}(L) \in C^{-}$. The eigenvalues can be chosen equal to the ones for the previous case. The, the gain matrix $N$ obtained is

$$
N=\left(\begin{array}{llll}
4.18 & 15.31 & 14.872 .19 & 1.56
\end{array}\right)^{T}
$$

\subsection{Simulation Results}

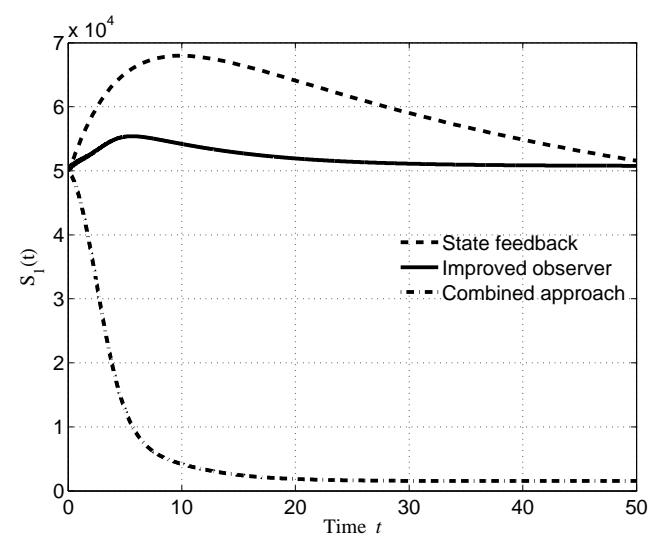

Fig. 1. Time history of individuals in $S_{1}(t)$.

Three cases have been simulated to compare their behaviours. The first is the direct use of the state feedback optimal control without the use of an observer. It is introduced as a benchmark for the other two approaches. This case in denoted 


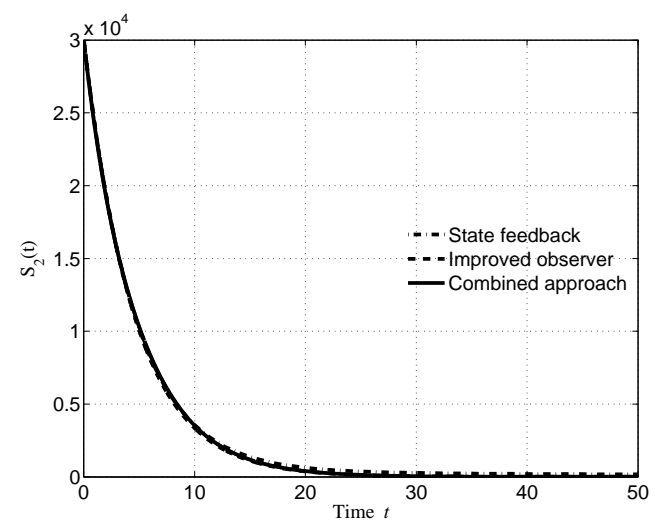

Fig. 2. Time history of individuals in $S_{2}(t)$.

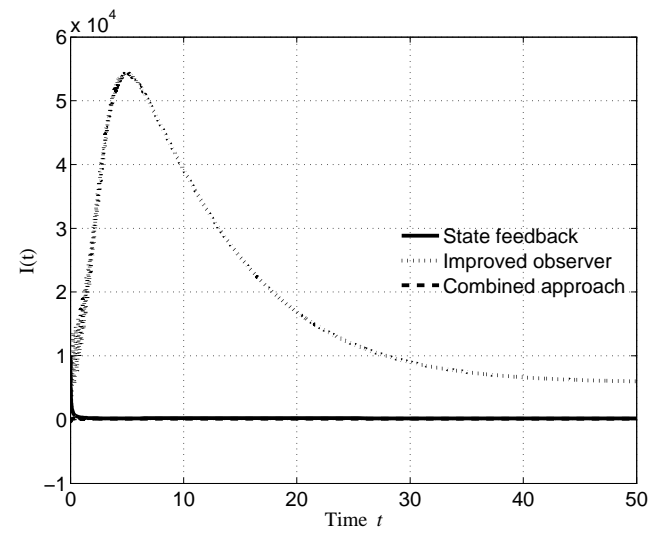

Fig. 3. Time history of individuals in $I(t)$.

in the legend of the Figures 1-5 by State feedback. Moreover, the two solutions described in this paper are reported in Figures 1-5; the approach described in Section 3 and numerically given in Subsection 6.1 is referred as Improved observer while the approach proposed in Section 4 with numerical values in Subsection 6.2 is addressed as Combined approach.

The pure state feedback control scheme, whose behaviour is reported in the Figures with a solid line, shows the effectiveness of the control strategy based on a local LQR problem solution: the number of infected $I$ is very quickly reduced to a very small value, Figure 3, and consequently, a decrease of the number of the diagnosed patients $P$, Figure 4 , and $A$, Figure 5 is obtained. At the same time, the number of healthy individuals $S_{1}$ is maintained sufficiently high, Figure 1, while, due to a reduction of the infection probability, the passage from $S_{1}$ to 


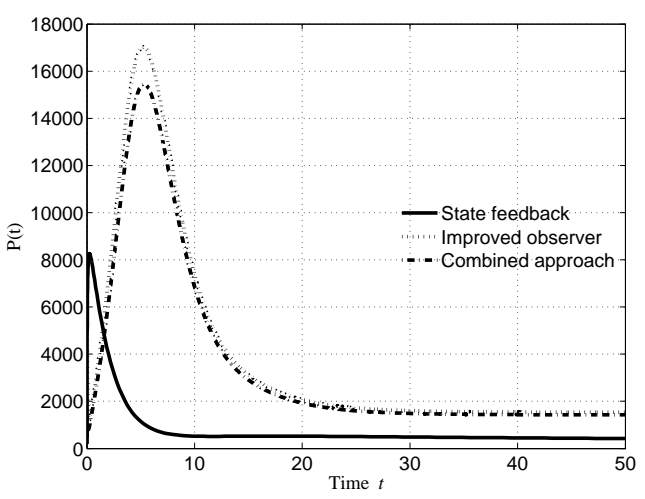

Fig. 4. Time history of individuals in $P(t)$.

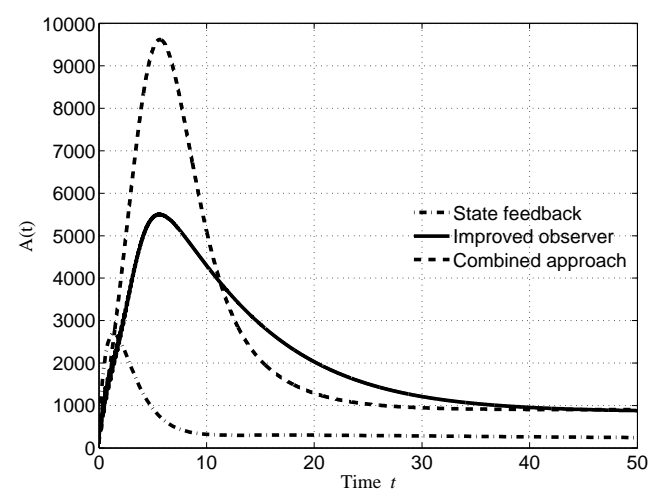

Fig. 5. Time history of individuals in $A(t)$.

$S_{2}$ is no more necessary for the spread containment and the individuals in $S_{2}$, reported in Figure 2, naturally tend to zero by natural death.

The effects of the two proposed approaches are also reported in the Figures: the dashed line, for which the denomination in the legend is Improved observer, depicts the behaviours of the state variables when the solution proposed in Section 3 for the observer design, with numerical values for the present case study reported in Subsection 6.1 is applied; the dash-dot line, marked with Combined approach, is devoted to depict the time histories of the state variables in the case of application of the procedure described in Section 4 and particularised to the present case study in Subsection 6.2.

The most evident effect in the use of a state observer, independently from which approach is followed in its design, is an expected different behaviour during the transient, since the control law bases its effect on a not well estimated state. When, asymptotically, the state estimation error goes to zero, the differences with the state feedback is strongly reduced. 
A comparative analysis of the performances of the two observer design procedures proposed put in evidence their differences at steady state, since the one which takes into account the actual working point shows a behaviour which tends to be similar to the one of the sate feedback. The other approach pays its design simplicity with an offset difference due to the fact that in this case the observer is designed to better approximate the state in a region that is different to the actual one according to the differences between the equilibrium point for the uncontrolled system and the one for the controlled dynamics.

\section{Conclusions}

This paper is an extension of the contribution presented at ICINCO2019. It discusses the problem of the implementation of a state feedback control, obtained solving a LQR problem for a linearised nonlinear dynamics, using a local asymptotic state observer. The approach proposed at ICINCO2019 is based on the construction of the local state observer which better approximates the dynamics in the neighbourhood of the final working point, supposed equal to the equilibrium of the state feedback controlled system. This result is here enriched starting from a different problem definition in which the linear state observer design is included in the LQR control problem and the local approximations are necessary performed in a neighbourhood of an equilibrium point of the initial uncontrolled system. The effectiveness of the two solutions obtained, as well as a comparison between them, are evidenced from the results of their implementation on a case study represented by a virus spread dynamics.

\section{References}

1. Anderson, B.D.O., Moore, J.B.: Optimal control (1989)

2. Andrieu, V., Praly, L.: On the existence of a Kazantis-Kravaris/Luenberger observer. SIAM Journal on Control and Optimization 45(2), 432-456 (2006)

3. Di Giamberardino, P., Compagnucci, L., Giorgi, C.D., Iacoviello, D.: A new model of the HIV/AIDS infection diffusion and analysis of the intervention effects. $25^{\text {th }}$ IEEE Mediterranean Conference on Control and Automation (2017)

4. Di Giamberardino, P., Compagnucci, L., Giorgi, C.D., Iacoviello, D.: Modeling the effects of prevention and early diagnosis on HIV/AIDS infection diffusion. IEEE Transactions on Systems, Man and Cybernetics: Systems (2018)

5. Di Giamberardino, P., Iacoviello, D.: LQ control design for the containment of the HIV/AIDS diffusion. Control Engineering Practice 77 (2018)

6. Di Giamberardino, P., Iacoviello, D.: An output feedback control with state estimation for the containment of the HIV/AIDS diffusion. Proceedings of the 26th Mediterranean Conference on Control and Automation (MED) (2018)

7. Di Giamberardino, P., Iacoviello, D.: An improvement in a local observer design for optimal state feedback control: The case study of hiv/aids diffusion. Proceedings of the 16th International Conference on Informatics in Control, Automation and Robotics (ICINCO2019) $\mathbf{1}$ (2019)

8. Kazantzis, N., Kravaris, C.: Nonlinear observer design using Lyapunov's auxiliary theorem. Proc. $36^{\text {th }}$ Conference on Decision \& Control (1997) 
9. Luenberger, D.G.: Observing the state of a linear system. IEEE Trans. on Military Electronics MIL-8(2), 74-80 (1964)

10. Massad, E.: A homogeneously mixing population model for the AIDS epidemic. Math. Comput. Modelling (1989)

11. Naresh, R., Tripathi, A., Sharma, D.: Modeling and analysis of the spread of AIDS epidemic with immigration of HIV infectives. Mathematical and Computer Modelling 49 (2009)

12. Respondek, W., Pogromsky, A., Nijmeijer, H.: Time scaling for observer design with linearizable error dynamics. Automatica 40 (2004)

13. Sassano, M., Astolfi, A.: A local separation principle via dynamic approximate feedback and observer linearization for a class of nonlinear systems. IEEE Trans. Aut. Control 64(1), 111-126 (2019)

14. Sundarapandian, V.: Local observer design for nonlinear systems. Mathematical and Computer Modelling 35 (2002)

15. Sundarapandian, V.: Reduced order observer design for nonlinear systems. Applied Mathematics Letters 19 (2006)

16. Zeitz, M.: The extended Luenberger observer for nonlinear systems. Syst. and Contr. Lett. 9 (1987) 